\title{
Asociación entre consumo de alcohol y relaciones sexuales ocasionales en los adolescentes
}

\author{
Ortiz- Melgar Mariela ${ }^{1, a}$, Pérez-Saavedra Vilma ${ }^{2, b}$, Valentín-Ballarta Jennifer J. 3,a, \\ Zúñiga- Hurtado Andrea L. 4,a
}

\begin{abstract}
RESÚMEN
Objetivos: determinar la relación entre consumo de alcohol y relaciones sexuales ocasionales en adolescentes de la Institución Educativa Nacional 6090 del distrito de Chorrillos - Lima, en el mes de abril 2011. Material y métodos: estudio descriptivo de corte transversal. La muestra estuvo constituida por 204 alumnos del 5to. Año, turnos mañana y tarde del nivel secundario, quienes cumplieron los criterios de inclusión. Los padres de familia firmaron el consentimiento informado, aceptando que sus hijos participen voluntariamente. Para la recolección de datos se utilizó como técnica la encuesta y como instrumento un cuestionario auto administrado, este fue anónimo. Respecto al procesamiento y análisis de la información se utilizó una base de datos creada en MS Excel y el análisis se realizó en SPSS versión 17; los resultados fueron presentados en cuadros estadísticos (simples y de doble entrada). Se utilizó la prueba de Chi cuadrado para probar la relación y asociación entre las variables cualitativas en estudio. Resultados: Mostraron que el $87,3 \%$ consumieron alcohol y el 83,8\% tuvieron relaciones sexuales ocasionales. De la relación de estas variables se obtuvo un $\mathrm{P}=0.000$, siendo el estudio significativo.Conclusiones: si existe relación entre el consumo de alcohol y relaciones sexuales ocasionales en los adolescentes.
\end{abstract}

PALABRAS CLAVE: Consumo de bebidas alcohólicas, conducta sexual, adolescentes y estudiantes.

\section{Association between alcohol and casual sex in the adolescents}

\section{SUMMARY}

Objectives: to determine the relationship between alcohol and casual sex among adolescents in the 6090 National Educational Institution district of Chorrillos - Lima in April 2011. Material and Methods: a descriptive study was cross-sectional. The sample consisted of 204 students of the 5th. Year, morning and afternoon shifts at the secondary level who met the inclusion criteria. It featured the participation of the parents who signed the informed consent, accepting their children to participate voluntarily. For data collection was used as a technique and as the survey questionnaire administered instrument car, this was anonymous. Regarding the processing and analysis of information a database created in MS Excel and the analysis was conducted in SPSS version 17 was used; the results were presented at (single and double entry) statistical tables. the chi-square test was used to test the relationship and association between qualitative variables under study. Results: showed that $87.3 \%$ consumed alcohol and $83.8 \%$ had casual sex. The relationship of these variables $\mathrm{P}=0.000$ was obtained, with the significant study. Conclusions: the correlation between alcohol and casual sex among adolescents.

KEYWORDS: Drinking, sexual behavior, teenagers and students.

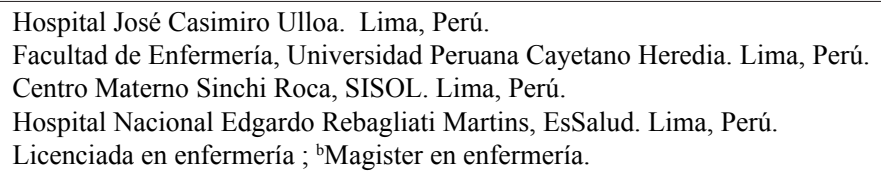




\section{INTRODUCCIÓN}

El consumo de alcohol es frecuente en adolescentes por diversos motivos como sentirse alegres por la euforia que produce al inicio del consumo, porque los hace sentir más seguros y les da más confianza facilitando la interacción grupal reduciendo la tensión psicológica (1); agregándose a estas motivaciones las características propias de la edad como el de conocer y experimentar nuevas sensaciones, lo que propicia el inicio de la vida sexual del adolescente bajo los efectos del alcohol.

La relación entre el consumo de alcohol y las relaciones sexuales ha sido estudiada por diversos autores como Ceballos quien encontró que los estudiantes que consumieron alcohol durante el último mes 85 (45,5\%) manifestaron haber tenido relaciones sexuales (2).

De Bedout encontró que el $(29,4 \%)$ de adolescentes manifiestan haber tenido relaciones sexuales bajo el efecto del licor (3). Díaz encontró que los factores individuales como el consumo de sustancias y la preferencia por actividades sociales están fuertemente relacionados con el inicio de su actividad sexual. Este hábito resultó ser el principal factor de riesgo para el inicio de la actividad sexual (OR: 7,11 AOR: 5,26). Una adolescente que consume bebidas alcohólicas tiene 5 a 7 veces más probabilidades de iniciar su actividad sexual que otra que no está expuesta a este hábito (4).

Por lo anteriormente mencionado y por la escasa información en el país respecto al tema es que se plantea estudiar la relación entre el consumo de alcohol y las relaciones sexuales ocasionales.

El objetivo del estudio fue determinar la relación entre consumo de alcohol y relaciones sexuales ocasionales en los adolescentes del nivel secundario de una Institución Educativa Nacional de Chorrillos, octubre-noviembre 2011- Lima Perú.

\section{MATERIAL Y METODOS}

El estudio fue descriptivo relacional de corte transversal, realizado en los meses de octubre a noviembre 2011 en una Institución Educativa estatal del distrito de Chorrillos en Lima - Perú.

Los participantes fueron estudiantes de ambos sexos de quinto año de secundaria de un colegio de Lima, Perú que tuvieron asistencia regular, de los turnos mañana y tarde,que aceptaron participar voluntariamente y que tu- vieron autorización de sus padres. La población estuvo constituido por el total de 204 estudiantes, 102 por turno.

Para la recolección de datos se utilizó un cuestionario auto administrado. El cuestionario recogió en su primera parte datos generales, la segunda parte tuvo 11 preguntas sobre el consumo de alcohol y práctica de relaciones sexuales ocasionales, fue validado por expertos, considerándose estadísticamente significativa con $\mathrm{P}<0,05$ en validez de contenido, de constructo y de criterio.Para su aplicación primeramente se explicó a los padres de familia y estudiantes el objetivo del estudio solicitándoles, a los primeros la autorización para la participación de sus hijos y a los estudiantes se les invito a participar en ambos casos su participación fue voluntaria. La aplicación de la encuesta se realizó en las aulas de clases de los estudiantesen el horario de tutoría, la duración fue de 20 minutos.

Los datos fueron ingresados en un base de datos diseñado en Excely los resultados fueron analizados en el programaestadístico SPSS versión 17. Se utilizó la prueba de Chi Cuadrado para probar la relación y asociación entre las variables en estudio.

El estudio fue revisado y aprobado por el Comité Institucional de Etica de la Universidad Peruana Cayetano Heredia.

\section{RESULTADOS}

Participaron en el estudio 204 estudiantes del quinto de secundaria, de los cuales el 81,4\% tenían entre 16 y 17 años de edad; y el 57,4\% fueron de sexo masculino.

Respecto al consumo de alcohol el 87,3\% de adolescentes manifestó haber consumido alcohol, y de estos el $76,4 \%$ manifestó haber iniciado su consumo en edades entre 13 y 15 años.

Otro aspecto estudiado fueron las relaciones sexuales ocasionales que tuvieron los estudiantes, 171 (83,8\%) manifestaron haber tenido relaciones sexuales ocasionales y de ellos $153(89,5 \%)$ manifestaron que iniciaron esta práctica sexual entre las edades de 14 a 16 años.

Al establecer la relación entre consumo de alcohol y relaciones sexuales ocasionales en adolescentes, se encontró una relación estadísticamente significativa con $\mathrm{p}=0,000$ obtenido mediante el Chi-Cuadrado (Tabla 1). 
Tabla 1. Relación entre consumo de alcohol y relaciones sexuales ocasionales en adolescentes de una Institución Educativa Nacional. Chorrillos, Lima, Perú.

\begin{tabular}{|c|c|c|c|c|}
\hline \multicolumn{2}{|c|}{$\begin{array}{l}\text { Consumo de } \\
\text { alcohol }\end{array}$} & \multicolumn{2}{|c|}{$\begin{array}{c}\text { Relaciones sexuales } \\
\text { ocasionales }\end{array}$} & \multirow[t]{2}{*}{ Total } \\
\hline & & Sí & No & \\
\hline & $\mathbf{N}$ & 170 & 8 & 178 \\
\hline Sí & $\%$ & $83,3 \%$ & $4,0 \%$ & $87,3 \%$ \\
\hline \multirow[t]{2}{*}{ No } & $\mathbf{N}$ & 1 & 25 & 26 \\
\hline & $\%$ & $0,5 \%$ & $12,2 \%$ & $12,7 \%$ \\
\hline \multirow[t]{2}{*}{ Total } & $\mathbf{N}$ & 171 & 33 & 204 \\
\hline & $\%$ & $83,8 \%$ & $16,2 \%$ & $100,0 \%$ \\
\hline
\end{tabular}

Chi-Cuadrado $=140,502 ; \mathrm{GL}=1 ; \mathrm{p}=0.000$ SIGNIFICATIVO

\section{DISCUSION}

El presente estudio comprende las variables consumo de alcohol, relaciones sexuales ocasionales y la relación entre estas, las cuales el $87,3 \%$ de los estudiantes que participó en el estudio manifestó haber consumido alcohol y su edad de inicio fue entre los trece y catorce años. Estos resultados son similares a los ya publicados en diferentes estudios que mencionaremos seguidamente:

Schaffer y col., encontraron que un 70,0\% de los jóvenes estudiantes consumían bebidas alcohólicas, y se habían iniciado en esta práctica entre 14 y 15 años de edad (5), igualmente Cuzcano y col., en su estudio "Consumo de alcohol y factores sociodemográficos en adolescentes de instrucción secundaria en Barrios Altos" encontraron que la edad de inicio de consumo de alcohol era a partir de los 14 años (6). Los resultados obtenidos en este estudio también son similares a los referidos por el Ministerio de Salud (MINSA) y DEVIDA que consideran que el inicio de consumo de alcohol es cada vez a menor edad, y se da a entre la edades de 11 a 14 años $(7,8)$.

El alto consumo de alcohol encontrado en los adolescentes del estudio, puede deberse a factores familiares, sociales y publicitarios y a las características propias de la adolescencia. Dentro de los factores familiares se considera el vivir con un solo padre, vivir con un padre bebedor, recibir maltrato físico, psicológico y/o sexual, mala relación familiar, bajos ingresos económicos, baja escolaridad del padre, ausencia de los padres por trabajo (9-12).

Agregándose a lo anteriormente mencionado están las características propias del adolescente que busca explorar y tener nuevas experiencias, ampliar su círculo social, en esta edad se inicia la asistencia a reuniones sociales, discotecas y bailes populares, lo que se convierte en una oportunidad para consumir alcohol (10-13).

Otro aspecto que influye en el consumo de alcohol a temprana edad es el fácil acceso que tienen los adolescentes para adquirir bebidas alcohólicas,por su bajo precio y expendio sin control a menores de edad, no obstante que está prohibido legalmente. Agregándose a ello están las campañas publicitarias en medios de comunicación, campañas deportivas etc. que tiene impacto al promover el consumo de bebidas alcohólicas en adolescentes. Esto es sustentado por distintos autores que concluyen que las campañas publicitarias, el patrocinio de equipos deportivos por compañías productoras de bebidas alcohólicas, el fácil acceso para adquirirlas están relacionados con el consumo de alcohol de los adolescentes $(12,14,15)$.

En relación a la variable relaciones sexuales ocasionales, la mayoría de adolescentes participantes en el estudio manifestó haber tenido relaciones sexuales ocasionales $(83,8 \%)$, y de ellos el $(89,5 \%)$ manifestaron que iniciaron esta práctica sexual entre las edades de 14 a 16 años. Estos resultados son similares a encontrados por Torriente, que reportó que cerca de $60 \%$ de adolescentes encuestados manifestaron haber tenido relaciones sexuales y que su edad de inicio fue antes de los 14 años (16).

Asimismo, los datos también coinciden a los hallados por Gamarra y col., quienes encontraron que un 38,9\% ya habían iniciado actividad sexual y el promedio de inicio de la primera relación sexual en ambos sexos fue de $14,58(16,17)$. Otro estudio que tiene datos coincidentes con el presente estudio es del Catacora-López y col., encontraron que el promedio de edad de inicio de las relaciones sexuales fue de 14,5 años en los hombres y 15,4 años en las mujeres (18). Otros estudios con resultados similares al presente estudio son los realizados en países como Costa Rica y Argentina como lo menciona Valverde, quien refiere que el inicio de las relaciones sexuales en adolescentes costarricenses se está dando a los 14 años y en algunos casos, a menor edad y el Ministerio de Salud de Argentina señala que los adolescentes se inician sexualmente antes de los 14 años $(19,20)$.

El inicio precoz de las relaciones sexuales en adolescente puede deberse a múltiples causas como, problemas en los ámbitos familiar, sentimental, social y académico. Los problemas familiares que pueden influir para el inicio precoz de relaciones sexuales en los adolescentes es vivir solos o con uno de los padres, la falta de co- 
municación y confianza de los padres para tratar temas de educación sexual, entre otros, lo cual es corroborado por Guerra y col., quienes refieren que el ámbito familiar ejerce decisiva influencia en la conducta sexual de los adolescentes, pues los jóvenes que viven en familias monoparentales (por lo general sin el padre), presentan mayor riesgo de iniciar su vida sexual a temprana edad (13 a 15 años), así como las hijas de madres adolescentes y aquellas que están menos integradas a su familia. La falta de confianza de los padres hacia sus hijos, para abordar temas sobre la sexualidad, obliga a los adolescentes a buscar esta información en quienes en su mayoría manejan información errada sobre estos temas (4, 21-23).

También pueden ser causas del inicio de relaciones sexuales tempranas la influencia de amigos y el bajo rendimiento académico en tal sentido Santana sostiene que si el grupo de pares se ha vinculado con la decisión de iniciar las relaciones sexuales, cuando los adolescente están más unidos al grupo y se comunican de forma activa parecen ser más proclives a iniciar las relaciones sexuales, sobre todo si los miembros del grupo al que pertenecen ya han iniciado su vida sexual (24). Los aspectos académicos también juegan un rol importante, pues los adolescentes que han repetido algún grado y tienen un rendimiento académico inferior o no tienen aspiraciones definidas, tienen mayor tendencia a iniciar las relaciones sexuales (24).

Se encontró relación significativa entre consumo de alcohol y las relaciones sexuales ocasionales en los adolescentes (Tabla 1). Resultados similares fueron hallados por De Bedout quien reportó que un $83,1 \%$ de adolescentes manifestaron haber consumido sustancias psicoactivas y de estos, un 51,3\% tuvieron relaciones sexuales la primera vez bajo el estado de alguna sustancia psicoactiva dentro de ellas se consideró al alcohol (3).

Asimismo, Arias y col., encontraron que un $18,4 \%$ de adolescentes relataron tener relaciones sexuales después del consumo de alcohol, y un $40,0 \%$ tuvieron relaciones sexuales con una persona poco conocida (25).

Por otro lado, Palacios y col., encontraron que la edad de la primera relación sexual se asoció positivamente con la edad de inicio del consumo de bebidas alcohólicas $(\mathrm{r}=$ $0,36, p<0,01)$. La frecuencia de las relaciones sexuales se relacionó con la frecuencia del consumo de alcohol ( $\mathrm{r}$ $=0,17, \mathrm{p}<0,01)$, la cantidad del consumo de alcohol $(\mathrm{r}=$ $0,18, p<0,01)$ y con el consumo excesivo del mismo ( $\mathrm{r}$ $=0,17, p<0,01)(26)$. Otros estudios en los que se encon- tró similares resultados la presente investigación fueron los hallados por Acevedo y col., quienes mencionan que al evaluar la relación entre el consumo de alcohol con el inicio o no de las relaciones sexuales encontraron que el consumo de alcohol entre los adolescentes se constituye en un factor de riesgo, un 34\% de adolescentes que estudiaron declararon haber mantenido su primera relación sexual bajo efectos del alcohol de estos el $22 \%$ y el $13,5 \%$ fueron varones y mujeres respectivamente (27).

De acuerdo a los resultados de los estudios anteriormente mencionados que coinciden con los del presente estudio, muestran la existencia de la relación entre el consumo de alcohol y las relaciones sexuales ocasionales en los adolescentes, probablemente estos resultados se deben a factores como, la falta de información, la baja autoestima y la mala relación familiar que explicaremos seguidamente:

La falta de información sobre el riesgo de embarazos no deseados y enfermedades de trasmisión sexual que implica tener relaciones sexuales ocasionales pueden estar influyendo para la práctica de relaciones sexuales ocasionales en los adolescentes de este estudio; puede agregarse a lo ya mencionado el desconocimiento de los efectos del alcohol y que al consumirlo el adolescente experimentará sensaciones de alegría y euforia llevándolo a tener una conducta compulsiva, permitiéndole entablar una relación social más fácil con personas desconocidas y así lograr un encuentro sexual ocasional. A lo expuesto se agregan las características propias de esta etapa, como el deseo de explorar y tener nuevas sensaciones, lo que conlleva a la curiosidad para realizar nuevas aventuras como las relaciones sexuales ocasionales (28).

Asimismo, la mala relación familiar la falta de comunicación de los padres sobre aspectos de la sexualidad de su hijo adolescente condiciona que esté busquen refugio y confíe en amistades que pueden influir en la toma de decisiones inadecuadas como el tener relaciones sexuales ocasionales bajo el efecto de alcohol, sin medir el riesgo de adquirir enfermedades de trasmisión sexual (29). Otro de los factores que puede estar influyendo en la relación de variables anteriormente descritas posiblemente es la baja autoestima de los adolescentes, lo cual es corroborado por Becoña y col., que sostienen que la baja autoestima explica que algunos jóvenes realicen consumos más altos de alcohol, mantengan relaciones sexuales con un mayor número de parejas y la no utilización de preservativos, dando como consecuencias enfermedades de transmisión sexual, embarazos no deseados y abortos (29). Se concluye que 
el consumo de alcohol está relacionado a practica de las relaciones sexuales ocasionales en adolescentes.

\section{REFERENCIAS BIBLIOGRAFICAS.}

1. Mora-Ríos J, Natera G. Expectativas, consumo de alcohol y problemas asociados en estudiantes universitarios de la ciudad de México. Revista Cubana de Medicina General Integral. 2001(citado 31 Julio 2009); 43(2): 8996. Disponible en: http://bvs.insp.mx/rsp/_files/File/2001/ VOL\%2043\%202/v43_2_expectativas.pdf

2. Ceballos G, Campo A. Prevalencia de relaciones sexuales en estudiantes de secundaria entre 10 y 12 años Santa Marta - Colombia. Rev fac med. 2006;54(1): 4-11 .

3. De Bedout A. Asociación entre consumo de sustancias y relaciones sexuales en adolescentes estudiantes en Vegachí, Antioquia, Colombia. Rev Psicol GEPU. 2010 (Citado Mayo 2011); 1 (2): 31-38. Disponible en: http://bibliotecadigital.univalle.edu.co/handle/ 10893/2231 ?mode $=$ full

4. Díaz T. Factores que inciden en el inicio de las relaciones sexuales en adolescentes. Ciencia y Salud virtual. 2010; 1(2):152-160.

5. Schaffer C, Arizaga V, Albarenque M, Haseitel B. Alcoholismo en la adolescencia. Revista de Posgrado de la VIa Cátedra de Medicina. 2004;139:24-28

6. Cuzcano S, Orellana T, Saenz M. Consumo de alcohol y factores sociodemográficos en adolescentes de las instituciones educativas estatales de instrucción secundaria de Barrios Altos. Tesis de Licenciatura. Lima, Perú: Universidad Peruana Cayetano Heredia; 2008.

7. Ministerio de Salud. Consumo de alcohol en niños y adolescentes, puede generar enfermedades, intoxicaciones y dependencia. ( internet).Lima, Perú: MINSA; 2010 (citado 2011 junio). Disponible en: http://www.minsa.gob. pe/portada/especiales/2010/embarazoadolescente/ archivos/noticia.pdf

8. DEVIDA. Adolescentes que consumen alcohol terminan en drogas. Lima, Perú: DEVIDA; 2011 (Citado julio 2011). Disponible en: http://radio.capital.com.pe/ menoressinalcohol/2011/04/28/devida-adolescentes-queconsumen-alcohol-terminan-en-drogas/

9. Soutullo C, Mardomingo M. Manual de Psiquiatría del Niño y del Adolescente. Madrid, España: Ed. Médica Panamericana; 2010. p .233.

10. Ramírez M, Andrade D. La familia y los factores de riesgo relacionados con el consumo de alcohol y tabaco en los niños y adolescentes (Guayaquil-Ecuador). Rev. Latino-Am. Enfermagem. 2005;13:813-818. (citado Julio 2011). Disponible en: http://www.scielo. b r / s c i e 1 o.p p p ? p i d = S 0104 $11692005000700008 \&$ script $=$ sci

11. Martinez Y. Centro de Investigación de la Población Africana. Infancias difíciles propician el consumo de alcohol en adolescentes. Tendencia21; 2010. (citado julio del 2011). Disponible en: http://www.tendencias21.net/
notes/Infancias-dificiles-propician-el-consumo-dealcohol-en-adolescentes_b2216137.html

12. Blas F, Chavesta C, Chiroque F. Alcohol y drogas: Una problemática social en estudiantes. Lima, Perú: Universidad San Porres; 2008. (Citado julio 2011). Disponible en: http://es.scribd.com/doc/6603250/Proyecto-deInvestigacion

13. Cicua D, Méndez M, Muñoz L, Factores en el consumo de alcohol en adolescentes. Pensamiento Psicológico. 2008;4(11): 115-134. (citado mayo 2011). Disponible en: http://portales.puj.edu.co/psicorevista/components/ com_joomlib/ebooks/PS11-7.pdf

14. Aparicio J. Uso y abuso de alcohol en escolares de nivel secundario de Lima: Li,a Perú: MINSA; 2002 (Citado mayo 2011). Disponible en: http://www.hhv.gob.pe/ revista/2002/usoabusooh.htm

15. Albarracin M, Muñoz L. Factores asociados al consumo de alcohol en estudiantes de los dos primeros años de carrera universitaria. LIBERABIT. 2008;14: 49-61. (citado Julio 2011). Disponible en: http://www.scielo.org. pe/pdf/liber/v14n14/a07v14n14.pdf

16. Torriente WN, Cedré A, Rodríguez JP. Conocimientos sobre la sexualidad en adolescentes de una secundaria básica. La Habana: Instituto Superior de Ciencias Médicas de La Habana, Facultad Calixto García Iñiguez; 20062007.

17. Gamarra-Tenorio P, Lannacone J. Factores asociados que influyen en el inicio de actividad sexual en adolecentes escolares de Villa San Francisco - Santa Anita, Lima-Perú-2009. The Biologist (Lima). 2010 (Citado mayo 2011); 8 (1): 54-72 Disponible en: http:// sisbib.unmsm.edu.pe/BVRevistas/biologist/v08_n1/pdf/ a06v8n1.pdf

18. Catacora-López F, Villanueva-Roque J. Conocimientos sobre VIH/SIDA y Prácticas sexuales de escolares de Tacna. Rev Peru med exp salud pública. 2007 (Citado Mayo 2010); 24(3): 240-247. Disponible en: $\quad$ http://www.scielo.org.pe/scielo.php?script=sci arttext\&pid $=$ S1726-46342007000300007

19. Valverde E. Relaciones sexuales en adolescentes. Costa Rica: Grupo Nación GN SA; 2008 (Citado Mayo 2011) Disponible en: http://wvw.nacion.com/ln_ee/2008/ octubre/07/opinion1728751.html

20. Roman V. El 23\% de los chicos tienen sexo antes de los 14 años. Buenos Aires: Arte Gráfico Editorial Argentino S.A; 2011 Disponible en: http://www.clarin.com/sociedad/ chicos-sexo-anos_0_489551167.html

21. Alegret J, Comellas J, Font P. Adolescentes: relaciones con los padres, drogas, sexualidad y culto al cuerpo. Barcelona. Editorial GRAO; 2005.p. 105.

22. Perinat A, Corral A, Crespo I, et al. Los adolescentes del siglo XXI: Un enfoque psicosocial. Barcelona: Editotrial UOC; 2003. Pág. 185

23. Guerra G. Inicio de las relaciones sexuales en adolescentes escolares de Ciudad de La Habana. La Habana, Cuba; 2008.

24. Santana F, Ovies G, Verdeja O. Características de la 
primera relación sexual en adolescentes escolares de Ciudad de La Habana. Rev Cub Sal Pub. 2006(Citado en julio 2011); 32(3):0-0. Disponible en: http://bvs.sld.cu/ revistas/spu/vol32_3_06/spu06306.htm\#cargo

25. Campo-Arias A, Ceballo G, Herazo E. Prevalencia de patrón de comportamiento de riesgo para la salud sexual y reproductiva en estudiantes adolescentes. Rev. LatinoAm Enfermagem 2010;18(2): 170-174. (Citado mayo 2011); Disponible en: http:/www.scielo.br/ scielo.php?pid=S0104-11692010000200005\&script=sci arttext\&tlng=es

26. Palacios J, Bravo M, Andrade P. Consumo de alcohol y conducta sexual de riesgo. Psychology International Appendix. 2007(Citado mayo 2011); 18 (4): 1-13 Disponible en: https://www.apa.org/international/ pi/2007/11/consumo.pdf

27. Acevedo M, Mitre A, Nahuel N. Factores asociados a la primera relacion sexual en las y los estudiantes que cursan 1 ero a 3 ero medio en el liceo Rodulfo Amando Phillipi. paillaco 2009. Tesis de Licenciatura. Paillaco, Chile: Universidad Austral; 2009. 101pp.

28. McDowell J, Hostetler B. Manual para consejeros de jóvenes. El Paso, Texas. Editorial Mundo Hispana; 2006. p. 181.
29. Becoña E, López-Durán A, Fernández E, Martínez U, Osorio J, Fraga J. Borracheras, conducción de vehículos y relaciones sexuales en jóvenes consumidores de cocaína y éxtasis. Rev. Adicciones; 2011 (citado agosto 2011); 23(3): 205-218 Disponible en: http://www.adicciones.es/ files/becona $\% 2023-3 . p d f$

\section{Correspondencia:}

Pérez-Saavedra Vilma

Correo electrónico: vilma.perez@upch.pe
Fecha de Recepción: 30 de setiembre del 2015.

Fecha de aceptación: 04 de noviembre del 2015. 